\title{
Polarimetric technique to study (pre)biological organics in cosmic dust and planetary aerosols
}

\author{
Lev Nagdimunov $^{1}$, Ludmilla Kolokolova ${ }^{1}$, and William Sparks ${ }^{2}$ \\ ${ }^{1}$ Department of Astronomy, University of Maryland, College Park, MD, USA \\ ${ }^{2}$ Space Telescope Science Institute, Baltimore, MD, USA
}

(Received October 31, 2012; Revised May 3, 2013; Accepted May 21, 2013; Online published October 24, 2013)

\begin{abstract}
Noticeable circular polarization was measured in several dusty environments, including star forming regions and comets. These measurements have raised the possibility that circular polarization may relate to the formation (in molecular clouds) and observation (in comets) of prebiological organics, characterized by homochirality (dominance of molecules of specific handiness). We present computer simulations of light scattering by aggregates to model circular polarization from realistic particles containing homochiral molecules. Using optical constants of chlorophyll $a$, we study the spectral and phase angle dependencies of circular polarization. We begin our study by reproducing laboratory measurements (Sparks et al., 2009a), which found a fast change and reversal of sign for circular polarization within absorption bands. We further study how this spectral effect depends on the size and number of monomers in aggregates. We show that larger aggregates are characterized by larger values of circular polarization and that circular polarization peaks at medium $\left(40-140^{\circ}\right)$ phase angles. We discuss at which wavelengths such a spectral feature can be expected for (pre)biological molecules other than chlorophyll. These findings may help observers to optimize spectral and geometrical parameters in the search of cosmic dust that contains prebiological organics or aerosols of biological origin in atmospheres of planets.
\end{abstract}

Key words: Light scattering, aggregates, circular polarization, homochirality, astrobiology.

\section{Introduction}

The detection of life in the universe is one of the driving forces behind astrobiology. A key component of a search for life in the universe is the identification of a signature unique to biological material. Many complex organic molecules exist in two mirror-symmetric forms, a dichotomy known as chirality. On Earth, biological molecules are typically found to be composed of only one of these two forms, e.g. "left-handed" amino acids or "right-handed" sugars. This preference, known as homochirality, is a potential signature of life because non-biological processes lead to equal (racemic) mixtures of right-handed and left-handed chiral constituents. Multiple authors have suggested that homochirality may be a universal property of life (Cohen, 1995; Toxvaerd, 2009), as it may be a necessity for selfreplication (Popa, 2004). It is already known that chirality is not a purely terrestrial phenomenon; excesses in left-handed amino acids have been found in meteorites (Cronin and Pizzarello, 1997; Pizzarello and Cronin, 2000). It has also been suggested that homochirality may explain circular polarization observed in comets (Rosenbush et al., 2007, 2008). Although the origin of homochirality is still not determined, there is evidence that it may be connected to early stages of the development of planetary systems, e.g. star forming regions, where homochiral organics may be produced by

Copyright (C) The Society of Geomagnetism and Earth, Planetary and Space Sciences (SGEPSS); The Seismological Society of Japan; The Volcanological Society of Japan; The Geodetic Society of Japan; The Japanese Society for Planetary Sciences; TERRAPUB.

doi:10.5047/eps.2013.05.012 asymmetric photolysis and asymmetric photochemical synthesis of the organic molecules in the dust particles (e.g., Hough et al., 2001).

A potential method to detect the presence of homochiral material remotely is to measure the circular polarization of the light it scatters. Electromagnetic radiation is composed of a magnetic and electric component, with most sources of such radiation containing more than a single atom. The direction of oscillation of the electric components from an ensemble of atoms is usually chaotic and uncorrelated, producing unpolarized radiation. However, in some cases the electric components of each electromagnetic wave oscillate in the same plane, producing linearly polarized radiation. In rare cases, the direction of the electric components continuously rotates as the wave travels, producing circularly polarized radiation. Electromagnetic waves passing through a material that contains molecules of a specific chirality (right handed or left handed) become partially circularly polarized, thus providing an opportunity to use circular polarization as an indication of the presence of homochiral molecules.

To use circular polarization as a tracer for homochirality, it is necessary to distinguish polarization caused by homochiral scattering from polarization due to other sources, such as the commonly occurring alignment of elongated particles by a magnetic field in dusty environments. Alignment situations are believed to be a source of circular polarization in molecular clouds, where high levels of circular polarization $(\sim 10 \%$ or more) have been measured (Bailey et al., 1998; Chrysostomou et al., 2000; Menard et 


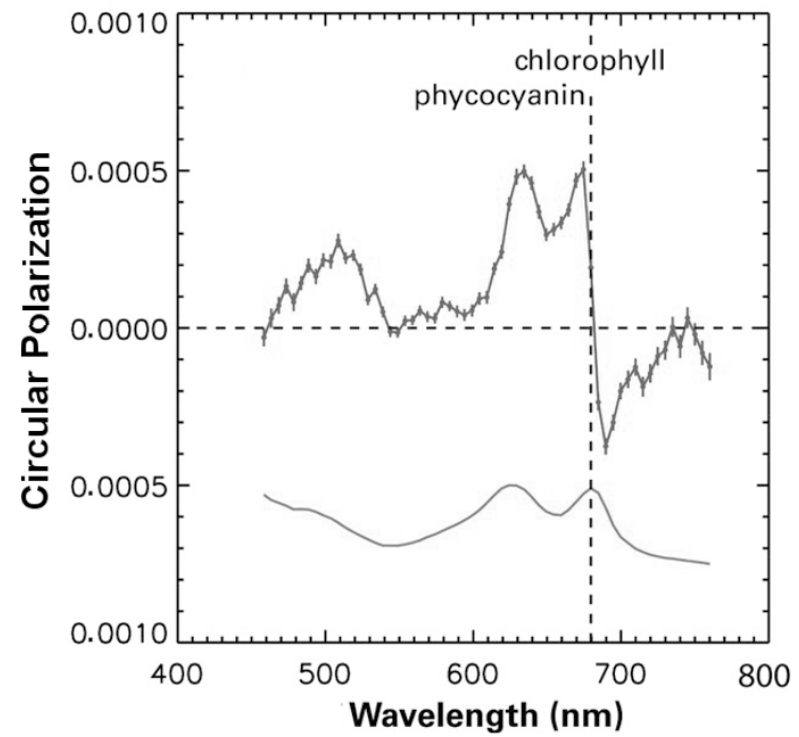

Fig. 1. Laboratory measurement of circular polarization spectra for cyanobacteria WH8101 shown at transmission (upper curve). Absorption shown in arbitrary units (lower curve). Adapted from Sparks et al. (2009a).

al., 1999). Laboratory experiments have demonstrated that circularly polarized incident radiation can both preferentially synthesize (via asymmetric photochemical synthesis) and destroy (via asymmetric photolysis) one form of chiral molecules, leaving the other to dominate (Balavoine et al., 1974; Flores et al., 1977; Norden, 1977; Greenberg et al., 1994; Meierhenrich and Thiemann, 2004; De Marcellus et al., 2011). This process could produce excesses in one form of chiral molecules during the protoplanetary stage, and spread to planets via impact events. As noted above, meteorites with a preference for left-handed amino acids have been found, and an amino acid (although achiralglycine) was recently found in comet 81P/Wild 2 (Elsila et al., 2009). Circular polarization measured on multiple comets has been hypothesized to stem from light scattering by chiral organic molecules or aggregates (Rosenbush et al., 2007, 2008). Although no circular polarization studies of exoplanets have been conducted to date, numerical modeling of polarization from exoplanets has already begun, including suggestions to look for homochiral organics (Karalidi et al., 2012).

Recent studies have shown that a key to identifying homochiral sources of circular polarization can be in the wavelength dependence of circular polarization (Pospergelis, 1969; Sparks et al., 2009a, b; Sterzik and Bagnulo, 2011). The spectral features that result from the homochiral nature of the organics are especially noticeable within strong absorption bands, which are typical in photosynthetic pigments. Circular polarization created by homochiral pigments was found to be not only non-zero but also to vary distinctly with wavelength inside absorption bands, showing a rapid change in value and in some cases even a change in sign. An example of circular polarization measurements is shown in Fig. 1 for cyanobacteria WH8101. Circular polarization shown in all figures of this paper is defined as the ratio of the forth Stokes parameter to the first one, i.e. V/I. Similar results were found for a variety of macroscopic vegetation and bacteria relying on photosynthesis for energy production (cyanobacteria). Control measurements of achiral chemical compounds and minerals showed either negligible circular polarization or noise-like variations of circular polarization not affected by a change in absorption (Sparks et al., 2009a, b).

The relationship between the absorption and the above described circular polarization spectral feature could allow future studies to distinguish between circular polarization in the light scattered by homochiral organics and polarization generated by other sources. Photosynthetic pigments can be of a special interest because photosynthesis is considered one of the most important biological processes on Earth. The early formation time and omnipresence of the pigments that allow for photosynthesis on Earth suggest that photosynthesis could exist elsewhere in the universe and thus allow for the detection of life outside of Earth.

In this paper, we present the results of computer modeling of circular polarization produced by light scattering of chlorophyll $a$ and compare them to the measurements of circular polarization (Sparks et al., 2009a, b) produced by cyanobacteria and vegetation, in which chlorophyll $a$ is abundant and essential. Chlorophyll $a$ is also a practical choice because its optical characteristics have been previously studied, whereas optical constants of other complex organics, e.g. amino acids, have been studied less extensively.

To advance both the study of circular polarization in photosynthetic pigments as well as circular polarization of homochiral molecules in general, we also performed a computational survey of the effect that properties of aggregates, used to simulate biological objects, have on circular polarization.

\section{Circular Polarization Induced by Homochiral Molecules}

Circular polarization of light scattered by homochiral molecules is caused by two material effects: optical rotation, sometimes also termed circular birefringence, and circular dichroism. Circular birefringence means that the material's real index of refraction is different for left and right circularly polarized light. Since unpolarized light can be described as the sum of equal amounts of right and left handed circularly polarized light, these two components of unpolarized light passing through a homochiral material will travel at different speeds. Similarly, circular dichroism, the other cause of circular polarization, refers to different absorption for light of different handedness. Both effects, in the absence of a magnetic field, arise only in particles characterized by an intrinsic asymmetry.

There are four optical constants necessary to model circular polarization of light that is scattered by a homochiral material. The first two are the real and imaginary parts of the complex index of refraction, describing refraction and absorption respectively. The other two parameters describe circular birefringence and circular dichroism. Mathematically, the complex index of refraction is $m=n+i k$, where $n$ is different for left and right-handed polarized light in the 
Table 1. Optical constants for chlorophyll $a$ used for light scattering calculations.

\begin{tabular}{ccccc}
\hline $\begin{array}{c}\text { Wavelength, } \\
\mathrm{nm}\end{array}$ & \multicolumn{2}{c}{$\begin{array}{c}\text { Complex refractive index } \\
(n)\end{array}$} & $\begin{array}{c}\text { Circular Birefringence } \\
(k)\end{array}$ & $\begin{array}{c}\text { Circular Dichroism } \\
\left(\beta_{I}\right)\end{array}$ \\
\hline 640 & 1.52 & $1.39 \mathrm{E}-03$ & $2.14 \mathrm{E}-08$ & $-5.54 \mathrm{E}-08$ \\
645 & 1.52 & $3.32 \mathrm{E}-03$ & $2.83 \mathrm{E}-08$ & $-5.77 \mathrm{E}-08$ \\
650 & 1.52 & $6.99 \mathrm{E}-03$ & $3.57 \mathrm{E}-08$ & $-6.19 \mathrm{E}-08$ \\
655 & 1.52 & $1.00 \mathrm{E}-02$ & $4.12 \mathrm{E}-08$ & $-7.28 \mathrm{E}-08$ \\
660 & 1.52 & $1.17 \mathrm{E}-02$ & $2.34 \mathrm{E}-08$ & $-7.85 \mathrm{E}-08$ \\
665 & 1.52 & $1.18 \mathrm{E}-02$ & $-1.24 \mathrm{E}-08$ & $1.70 \mathrm{E}-08$ \\
670 & 1.52 & $7.30 \mathrm{E}-03$ & $-1.18 \mathrm{E}-08$ & $2.27 \mathrm{E}-07$ \\
675 & 1.52 & $3.18 \mathrm{E}-03$ & $-7.13 \mathrm{E}-09$ & $3.62 \mathrm{E}-07$ \\
680 & 1.52 & $9.00 \mathrm{E}-04$ & $-2.56 \mathrm{E}-09$ & $3.54 \mathrm{E}-07$ \\
690 & 1.52 & $1.00 \mathrm{E}-06$ & $3.38 \mathrm{E}-09$ & $1.21 \mathrm{E}-07$ \\
\hline
\end{tabular}

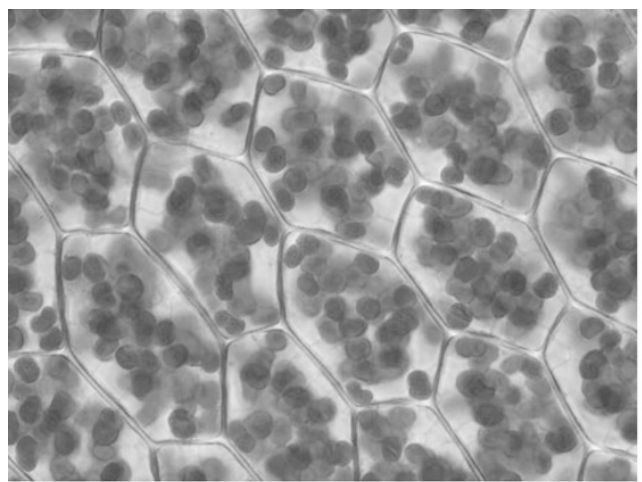

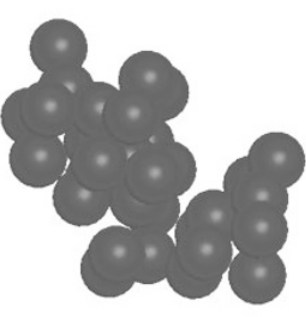

BPCA

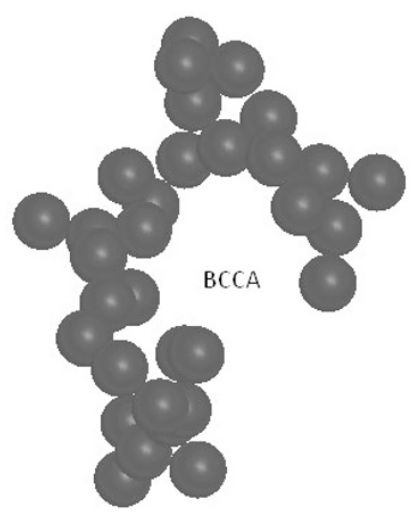

Fig. 2. Chloroplasts visible in the cells of a Plagiomnium affine plant (credit K. Peters. http://commons.wikimedia.org/wiki/File:Plagiomnium_ affine_laminazellen.jpeg), left, and two typical BPCA and BCCA aggregates used in our simulation, right. Note the similarity between the Chloroplast and BPCA structures.

presence of circular birefringence and $k$ is different in the presence of circular dichroism. We can create a parameter $\beta$, which describes the difference in the complex index of refraction between left and right-handed circularly polarized light: $\beta=m_{L}-m_{R}=\beta_{R}+i \beta_{I}$, where $\beta_{R}$ represents circular birefringence, $\beta_{I}$ represents circular dichroism. If we suppose that $m_{L}=\left(n+\frac{\beta_{R}}{2}\right)+i\left(k+\frac{\beta_{I}}{2}\right)$ describes the optical characteristics of left-handed light, then $m_{R}=\left(n-\frac{\beta_{R}}{2}\right)+i\left(k-\frac{\beta_{I}}{2}\right)$ describes right-hand circularly polarized light. Optical constants of chlorophyll $a$ used for our computations were extracted from Sauer (1965), Houssier and Sauer (1970), and Aas (1996) and adjusted for the chlorophyll concentration typical of cyanobacteria, $5^{*} 10^{-4} \mathrm{~mol} / \mathrm{L}$ (Satoh et al., 2001). These adjusted optical constants are specified in Table 1 as a function of wavelength.

\section{Modeling with Aggregates}

We use aggregates composed of multiple constituent particles to model biological material due to the prevalence of aggregate structures in nature and because the chlorophyll molecule is typically contained inside aggregated structures such as chloroplasts (Fig. 2). Aggregates of chloroplasts are found in plant cells to contain numerous membrane-bound compartments (known as thylakoids), which are the primary stores of light absorbing pigments such as chlorophyll $a$. Thylakoid membranes are also found in cyanobacteria, once again being the primary store of photosynthetic pigments.
Two simple aggregation-accretion algorithms were used to generate aggregates used in our simulations; the algorithms build the ballistic particle-cluster aggregate (BPCA) and the ballistic cluster-cluster aggregate (BCCA) (Meakin, 1983). It has recently become common to refer to constituent particles as "monomers" and use "particle" for the whole dust particle, aggregated or otherwise. We will follow this convention in the paper.

BPCA models are formed by starting with one monomer and then considering a new monomer, which begins at a random position and has a random velocity. If the new monomer intersects with any part of the original monomer, it sticks, creating a two monomer particle (cluster). The particle is then grown by repeatedly adding a new monomer one at a time until the desired number of monomers is reached. Each monomer is of the same size. The resulting porosity of the particle is typically between $82 \%$ and $88 \%$, where the porosity is defined as a ratio of the volume of the voids in the particle to the total volume of the particle (Mukai et al., 1992).

BCCA models initially create two particles using the BPCA model, where each particle is only composed of two monomers. These particles then collide to form one fourmonomer particle. The process then repeats from the beginning to form another four monomer particle, which is then collided with the already formed four monomer particle to create an eight monomer cluster. The process repeats until a desired number of monomers in the particle is reached. 
Particles formed via the BCCA model are less compact than those formed via the BPCA model, with typical porosities ranging from $92 \%$ to $99.9 \%$. A majority of our simulations utilized the BPCA model because of its larger similarity to Chloroplasts as compared to the BCCA model (Fig. 2). In our study on porosity, however, we found that the results for the BCCA model are quite similar and easily convertible qualitatively.

Light scattered by a single ballistic aggregate, even if the material was not homochiral, would have a circular polarization even if its orientation is averaged because the aggregate itself is asymmetric; this is another way of producing circularly polarized light (Kolokolova et al., 2006; Guirado et al., 2007). However, in real world situations, the multitude of aggregates with different structures would cancel each other's circular polarization if homochiral materials are not involved. In the case of homochiral materials, considering one specific aggregate would affect the value of circular polarization as the effects of homochirality and aggregate's asymmetry are combined. In our computer simulations we correct for this effect by calculating the polarization of two aggregates: the original aggregate and its mirror image. We then add the resultant Stokes parameters, producing the circular polarization that comes only from the difference in the indices of refraction, through which homochirality is reflected in the simulation. When we set optical rotation and circular dichroism to zero, the resultant circular polarization became zero as expected (Mackowski et al., 2011).

In order to calculate the circular polarization of light that has been scattered by intrinsically asymmetric (homochiral) particles, we utilized publically available paralleled Multiple Sphere T-matrix (MSTM) code developed and maintained by Daniel Mackowski at http://eng.auburn.edu/users/dmckwski/scatcodes/. MSTM was developed for simulating light scattering by a cluster of homogeneous spheres (Mackowski and Mishchenko, 1996, 2011), and was then modified to be able to simulate chiral optical qualities (Mackowski et al., 2011). Under this study, modifications of MSTM were performed to enable the computation of aggregate and mirror image during one computer run and thereby preserve precision during the addition of Stokes parameters.

\section{Results of Computer Simulations}

Light scattering computations were done for the wavelength range of $640 \mathrm{~nm}$ to $690 \mathrm{~nm}$, a region where chlorophyll $a$ has a large absorption band. Figure 3 demonstrates circular polarization that results from light scattering by aggregates composed of homochiral chlorophyll $a$. The resemblance to the laboratory measurements of cyanobacteria and macroscopic vegetation (Fig. 1) is clearly seen. This is an expected result because both cyanobacteria and macroscopic vegetation contain large amounts of chlorophyll, whose optical properties are responsible for the spectral behavior of circular polarization.

The most exciting feature of the laboratory and computer data is a change in sign of circular polarization that happens at the wavelength near largest absorption. This phenomenon is associated with Cotton effect (Cotton, 1895;

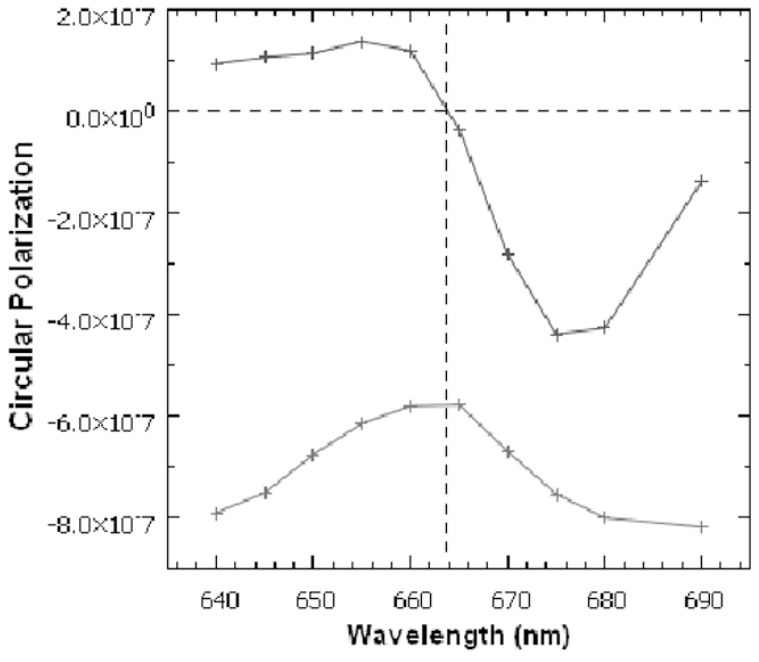

Fig. 3. Simulated spectral dependence of circular polarization shown at transmission (upper curve). Absorption shown in arbitrary units (lower curve). The aggregate contains 256 monomers of radius 0.1 micron.

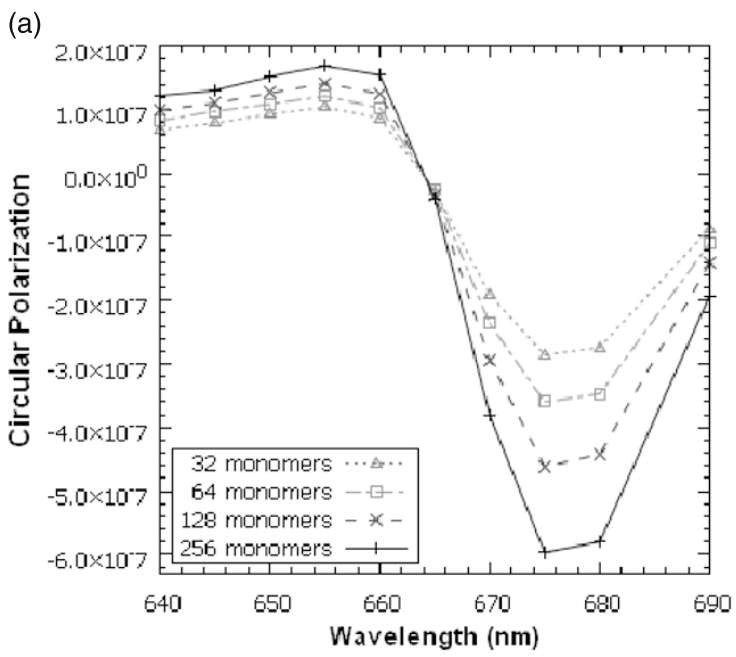

(b)

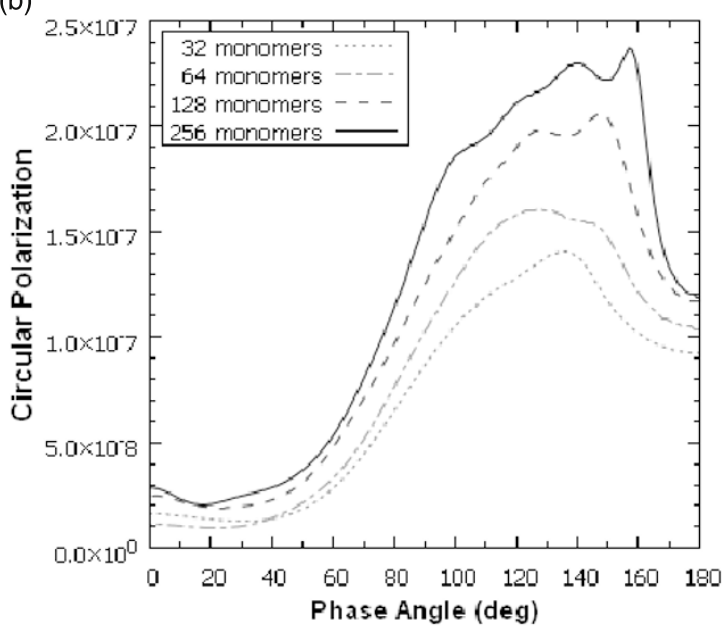

Fig. 4. Dependence of circular polarization on the number of the monomers as a function of (a) wavelength and (b) phase angle. The number of monomers, each of radius 0.1 micron, is specified in the legend. Spectral dependence, (a), is shown at phase angle $90^{\circ}$. Wavelength for (b) is $660 \mathrm{~nm}$. 
Eliel et al., 1994), where the circular dichroism and/or optical rotatory dispersion change sign through an electronic absorption band.

Although the simulated trend of circular polarization spectra is well matched to the laboratory data and suggests that our model correctly reproduces polarization trends, the magnitude of the modeled results is several orders smaller than measured in the lab. To determine whether our model could eventually reproduce the circular polarization magnitude, to study how stable the results are with changing parameters, and to look for other possible polarization trends that might later be helpful in using homochirality as a biosignature we then investigated how the properties of the aggregates affect the results.

Number of Constituent Monomers: Through additional modeling, we found that increasing the number of monomers forming an aggregate produces an increased magnitude of circular polarization. Figure 4(a) shows the spectral behavior of computed forward scattering (transmission) circular polarization within the absorption band of the chlorophyll. Note the larger values of circular polarization for larger aggregates. This is the result of two factors: (1) the larger amount of homochiral material in bigger aggregates and (2) more efficient electromagnetic interaction between monomers. The influence of electromagnetic interaction on polarization has been repeatedly emphasized in studies of aggregated particles (see Kolokolova and Kimura, 2010, and references therein). Electromagnetic interaction can roughly be described as multiple scattering in the aggregate and behaves similarly to multiple scattering in asymmetric media (e.g. Whitney and Wolff, 2002), intensifying circular polarization produced by a single optically active monomer. Large aggregates may be compared to optically thick media: incoming radiation is more likely to be held up inside the aggregate more efficiently, increasing chances for electromagnetic interaction between the monomers. We anticipate that by increasing the number of monomers in each single aggregate, and, possibly, including multiple scattering between different aggregates, we could reproduce the magnitude of circular polarization measured in the laboratory, although this task is very computationally intensive.

We also examined the dependence of polarization trends on the geometry of light scattering by changing the phase angle (source-aggregate-observer angle). With an increased number of monomers in the aggregate, the magnitude of circular polarization increases across all phase angles, i.e. for all geometrical situations (Fig. 4(b)), although the overall shape of the curve remains unaffected.

Size of Monomers: Figure 5 shows that circular polarization increases for larger size of monomers. This is typical for both the spectral behavior (Fig. 5(a)) and for most phase angles (Fig. 5(b)). The fact that increasing the size of the monomers results in increasing circular polarization is an expected result since it provides a larger amount of homochiral material for light to interact with. The plots for monomers of radius $\geq 0.7$ micron show an oscillating behavior in circular polarization, with a decreased magnitude of circular polarization in forward and back scattering regions. This effect is due to the monomer sizes being equal
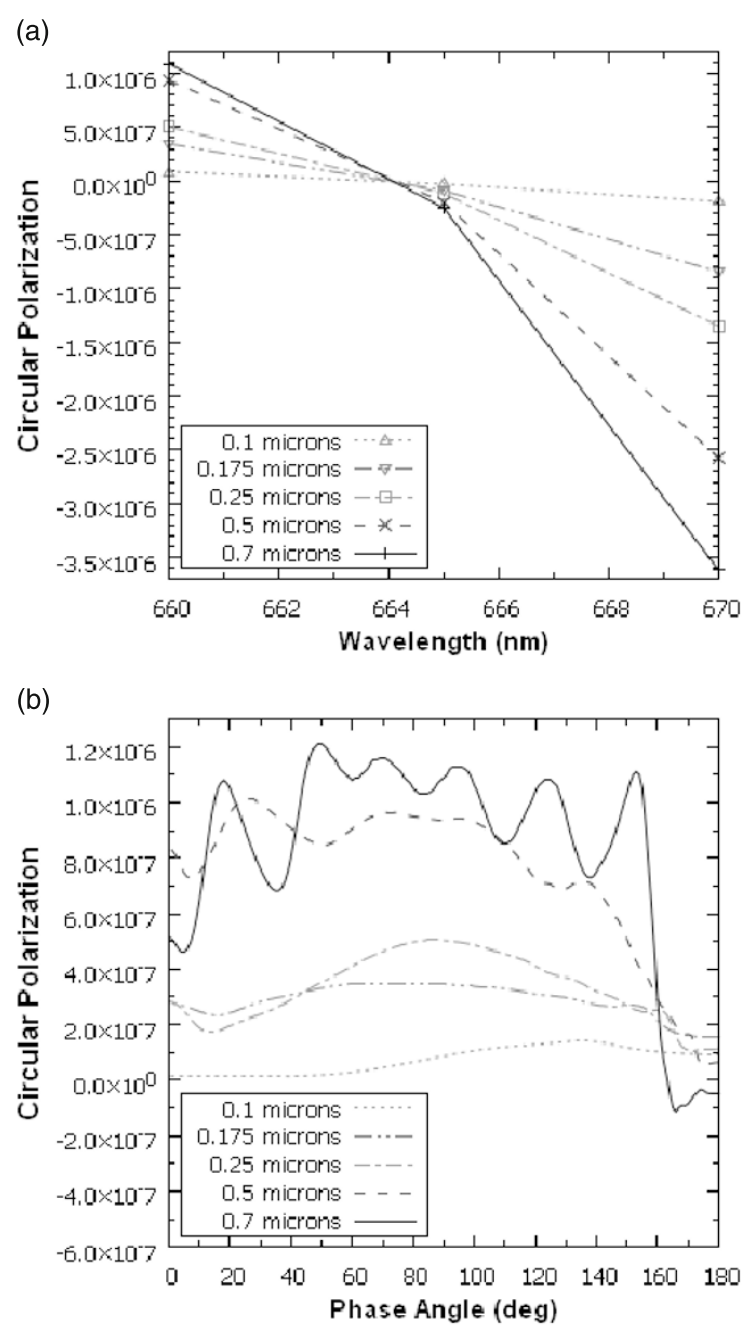

Fig. 5. Dependence of circular polarization on the size of the monomers as a function of (a) wavelength and (b) phase angle. Each aggregate contains 32 monomers, radii are given in microns at the bottom of the figure. Spectral dependence, (a), is shown at phase angle $90^{\circ}$ for the range of wavelengths $660-670 \mathrm{~nm}$. Wavelength for (b) is $660 \mathrm{~nm}$.

to or larger than the wavelength of incoming light. This, especially for small aggregates, results in the fact that the electromagnetic interaction between the monomers is weak and the characteristics of a single monomer dominate light scattering. Similar behavior can be seen in the results for linear polarization by Shen et al. (2009) who noticed that the oscillations will disappear if one considers monomers of nonspherical shape or aggregates with a larger number of monomers.

Interestingly, circular polarization peaks near intermediate phase angles; this suggests that it may be advisable for future observations to focus there.

Porosity: Using the two aggregate models, BPCA and BCCA, we determined how the circular polarization depends on the compactness of the aggregate. For these simulations, the size of the monomers and the size of the aggregate were kept identical to ensure that the amount of homochiral material did not change, only porosity varied. The results show that more compact aggregates polarize light more than their less compact counterparts (Fig. 6). This result is expected since the strength of electromagnetic inter- 
(a)

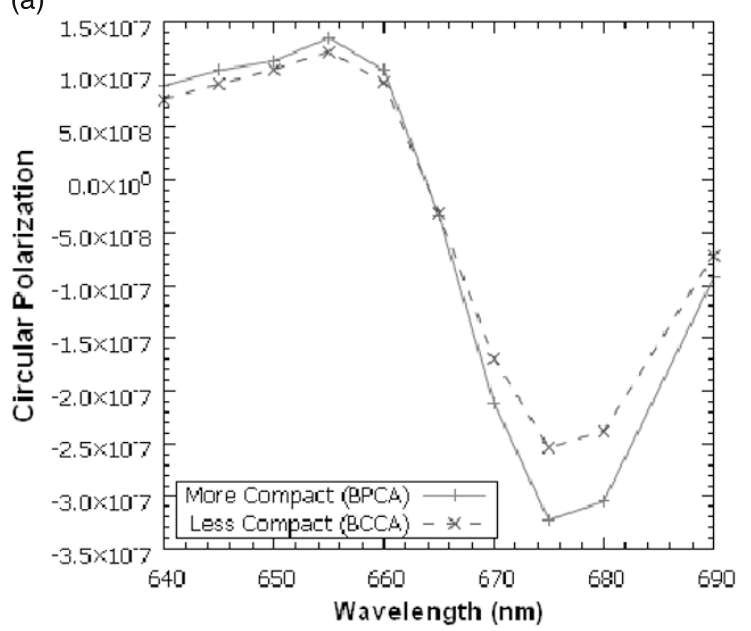

(b)

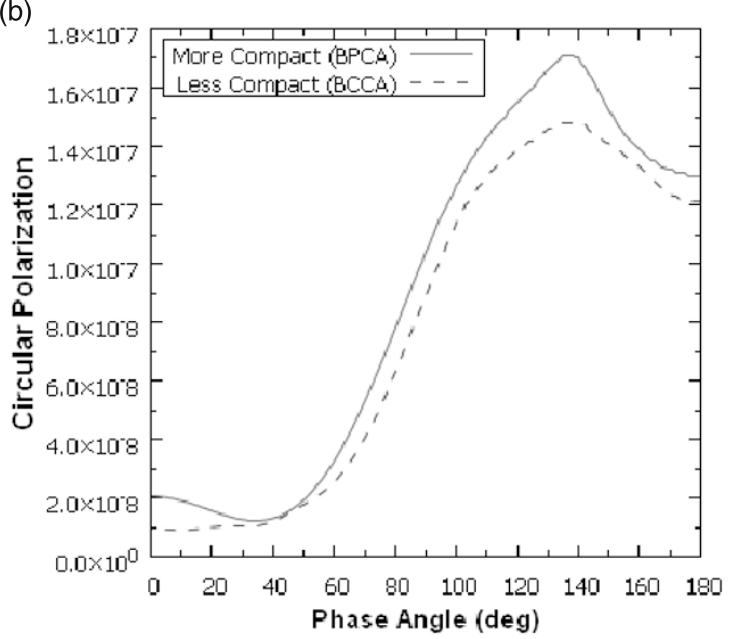

Fig. 6. Dependence of circular polarization on the porosity presented for BPCA and BCCA aggregates: (a) spectral dependence shown at transmission, and (b) phase-angle dependence at the wavelength 660 $\mathrm{nm}$. Each aggregate contains 32 monomers of radius 0.1 micron.

action depends on the number of monomers that are covered by a single wavelength (Kolokolova and Kimura, 2010); in a porous aggregate a high probability exists that this number is smaller than in the case of a compact aggregate. The shape of the polarization curve is not significantly altered with porosity as a function of either wavelength or phase angle, which indicates that our previous results, done primarily for BPCA, also apply to BCCA.

\section{Conclusions}

We utilized circular polarization previously measured for cyanobacteria and vegetation to show that our computer simulation methodology can reproduce the correct spectra of circular polarization introduced by homochiral pigments. As first observed in laboratory measurements of cyanobacteria and other photosynthetic organisms, a large change in the value and even sign of circular polarization in our simulations occurs within the absorption band of the material.

We have found that this effect, although produced by optical activity resulting from specific electronic transitions in chlorophyll molecules, depends on the characteristics of the scattering object. If we present the object as an aggregate, then the size of the monomers, their number in aggregate, and aggregate's porosity play a significant role. This shows that circular polarization can be a valuable tool not only to detect (pre)biological materials in space but also to reveal the size and structure of the objects that produce circular polarization.

Computer simulations demonstrate a clear trend that increasing the size or compactness of an aggregate produces larger values of circular polarization. Our current simulations were not intended and not able to reproduce the magnitude of measured circular polarization. This was partially due to the small number and size of monomers we had to use because of the large computational power required to run the calculations and partially because the lab measurements dealt with optically thick media where multiple scattering between the particles increased the values of circular polarization. Nevertheless, the trends we obtained clearly indicate that larger aggregates are necessary to produce larger values of circular polarization.

While our research focused primarily on photosynthetic pigments, we expect similar results may occur in other homochiral organics. Multiple amino acids have been found to show optical characteristics akin to chlorophyll $a$ in both circular birefringence and dichroic spectra around a wavelength of 200 nanometers (Iizuka and Yang, 1964; Hawkins and Lawrance, 1973; Meierhenrich et al., 2010). We thus suggest that observations of circular polarization spectra around absorption bands of amino acids may allow for their remote detection in comets, and potentially other objects that may contain (pre)biological organics.

Our modeling not only adds weight to the plausibility of using circular polarization to detect life or its precursors but also indicates some consequences for observers. In particular, photosynthesis bands that have the strongest polarization signatures are, most likely, associated with the wavelengths of maximum emission for the central star. Thus, the spectral range of maximum emission can be recommended for circular polarization measurements in the search for life on exoplanets. We also found that circular polarization increases with phase angle, reaching the highest values at phase angles $40-140^{\circ}$. This, too, is fortuitous since this is the most probable angles for future observations of exoplanets seen by direct imaging, and is well-matched to the phase angles needed to characterize any organic aerosols in their atmospheres.

Acknowledgments. This work has been supported by grant \#NNX09AM97G from the NASA Astrobiology Program. We thank Daniel Mackowski for the light scattering code used in our simulations. L. Kolokolova acknowledges the travel grant that allowed attending Cosmic Dust V meeting.

\section{References}

Aas, E., Refractive index of phytoplankton derived from its metabolite composition, J. Plankton Res., 18, 2223-2249, 1996.

Bailey, J., A. Chrysostomou, J. H. Hough, T. M. Gledhill, A. McCall, S. Clark, F. Menard, and M. Tamura, Circular polarization in starformation regions: Implications for biomolecular homochirality, Science, 281, 672-674, 1998.

Balavoine, G., A. Moradpour, and H. B. Kagan, Preparation of chiral compounds with high optical purity by irradiation with circularly polarized light, a model reaction for the prebiotic generation of optical activity, $J$. Am. Chem. Soc., 96, 5152-5158, 1974. 
Chrysostomou, A., T. M. Gledhill, F. Ménard, J. H. Hough, M. Tamura, and J. Bailey, Polarimetry of young stellar objects-III. Circular polarimetry of OMC-1, Mon. Not. R. Astron. Soc., 312, 103-115, 2000.

Cohen, J., Getting all turned around over the origins of life on Earth, Science, 267, 1265-1266, 1995.

Cotton, A., Anomalous rotatory dispersion in absorbing bodies, $C$. R. Acad. Sci., 120, 1044-1046, 1895 (in French).

Cronin, J. R. and S. Pizzarello, Enantiomeric excesses in meteoritic amino acids, Science, 275, 951-955, 1997.

De Marcellus, P., C. Meinert, M. Nuevo, J.-J. Filippi, G. Danger, D. Deboffle, L. Nahon, L. Le Sergeant d'Hendecourt, and U. J. Meierhenrich, Non-racemic amino acid production by ultraviolet irradiation of achiral interstellar ice analogs with circularly polarized light, Astrophys. J. Lett., 727, L27, 2011.

Eliel, E. L., S. H. Wilen, and L. N. Mander, Stereochemistry of Organic Compounds, Wiley, New York, 1994.

Elsila, J. E., D. P. Glavin, and J. P. Dworkin, Cometary glycine detected in samples returned by Stardust, Meteorit. Planet. Sci, 44, 1323-1330, 2009.

Flores, J. J., W. A. Bonner, and G. A. Massey, Asymmetric photolysis of (RS)-leucine with circularly polarized ultraviolet light, J. Am. Chem. Soc., 99, 3622-3625, 1977.

Greenberg, J. M., A. Kouchi, W. Noessem, H. Irth, J. Paradijs, M. Groot, and W. Hermsen, Interstellar dust, chirality, comets and the origins of life: Life from dead stars?, J. Biol. Phys., 20, 61-70, 1994.

Guirado, D., J. W. Hovenier, and F. Moreno, Circular polarization of light scattered by asymmetrical particles, J. Quant. Spectr. Radiat. Transfer, 106, 63-73, 2007.

Hawkins, C. L. and G. A. Lawrance, Circular dichroism spectra of N,NDimethyl-L-amino acids, Aust. J. Chem., 26, 1801-1803, 1973.

Hough, J. H., J. A. Bailey, A. Chrysostomou, T. M. Gledhill, P. W. Lucas, M. Tamura, S. Clark, J. Yates, and F. Menard, Circular polarization in star-forming regions: possible implications for homochirality, $A d v$. Space Res., 27, 313-322, 2001.

Houssier, C. and K. J. Sauer, Circular dichroism and magnetic circular dichroism of the chlorophyll and protochlorophyll pigments, Am. Chem. Soc., 4, 779-791, 1970.

Iizuka, E. and J. T. Yang, Optical rotatory dispersion of L-amino acids in acid solution, Biochemistry, 3, 1519-1524, 1964.

Karalidi, T., D. M. Stam, F. Snik, S. Bagnulo, W. B. Sparks, and C. U. Keller, Observing the Earth as an exoplanet with LOUPE, the Lunar Observatory for Unresolved Polarimetry of Earth, Planet. Space Sci., 74, 202-207, 2012.

Kolokolova, L., H. Kimura, K. Ziegler, and I. Mann, Light-scattering properties of random-oriented aggregates: Do they represent the properties of an ensemble of aggregates?, J. Quant. Spectr. Radiat. Transfer, 100, 199-206, 2006.

Kolokolova, L. and H. Kimura, Effects of electromagnetic interaction in the polarization of light scattered by cometary and other types of cosmic dust, Astron. Astrophys., 513, 513-517, 2010.

Kolokolova, L., E. Petrova, and H. Kimura, Effects of interaction of electromagnetic waves in complex particles, in Electromagnetic Waves, edited by V. Zhurbenko, 173-202, InTech, Vienna, 2011.

Mackowski, D. W. and M. I. Mishchenko, Calculation of the T matrix and the scattering matrix for ensembles of spheres, J. Opt. Soc. Am. A, 13, 2266-2278, 1996.

Mackowski, D. and M. I. Mishchenko, A multiple sphere T-matrix Fortran code for use on parallel computer clusters, J. Quant. Spectr. Radiat. Transfer, 112, 2182-2192, 2011.

Mackowski, D., L. Kolokolova, and W. Sparks, T-matrix approach to calculating circular polarization of aggregates made of optically active materials, J. Quant. Spectr. Radiat. Transfer, 112, 1726-1732, 2011.

Meakin, P., Formation of fractal clusters and networks by irreversible diffusion-limited aggregation, Phys. Rev. Lett., 51, 1119-1122, 1983. Meierhenrich, U. J. and W. H. Thiemann, Photochemical concepts on the origin of biomolecular asymmetry, Origins Life Evol. B, 34, 111-121, 2004.

Meierhenrich, U. J., J.-J. Filippi, C. Meinert, J. H. Bredehöft, J.-i. Takahashi, L. Nahon, N. C. Jones, and S. V. Hoffmann, Circular dichroism of amino acids in the vacuum-ultraviolet region, Angew. Chem. Int. Ed., 49, 7799-7802, 2010.

Menard, F., A. Chrysostomou, T. M. Gledhill, J. H. Hough, and J. Bailey, High circular polarization in the star forming region NGC 6334: Implications, Bioastronomy 99: A New Era in the Search for Life, 355-358, 1999.

Mukai, T., H. Ishimoto, T. Kozasca, J. Blum, and J. M. Greenberg, Radiation pressure forces of fluffy porous grains, Astron. Astrophys., 262, 315-320, 1992.

Norden, B., Was photoresolution of amino acids the origin of optical activity in life?, Nature, 266, 567-568, 1977.

Pizzarello, S. and J. R. Cronin, Non-racemic amino acids in the Murray and Murchison meteorites, Geochim. Cosmochim. Acta, 64, 329-338, 2000.

Popa, R., Between Necessity and Probability: Searching for the Definition and Origin of Life, Springer, Berlin, 2004.

Pospergelis, M. M., Spectroscopic measurements of the four Stokes parameters for light scattered by natural objects, Sov. Phys. Astron., 12, 973-977, 1969.

Rosenbush, V., L. Kolokolova, A. Lazarian, N. Shakhovskoy, and N. Kiselev, Circular polarization in comets: Observations of Comet C/1999 S4 (LINEAR) and tentative interpretation, Icarus, 186, 317-330, 2007.

Rosenbush, V., N. Kiselev, and L. Kolokolova, Predominantly left-handed circular polarization in comets: Does it indicate L-enantiomeric excess in cometary organics?, Proc. IAU, IAU Symposium, 251, 311-312, 2008. Satoh, S., M. Ikeuchi, M. Mimuro, and A. Tanaka, Chlorophyll b expressed in Cyanobacteria functions as a light-harvesting antenna in photosystem I through flexibility of the proteins, J. Biol. Chem., 276, 4293-4297, 2001.

Sauer, K., Optical rotatory dispersion of chlorophyll in solution and in chloroplast subunits, Proc. Natl. Acad. Sci., 53, 716-720, 1965.

Shen, Y., B. T. Draine, and E. T. Johnson, Modeling porous dust grains with ballistic aggregates. II. Light scattering properties, Astrophys. J., 696, 2126-2137, 2009.

Sparks, W. B., J. H. Hough, T. A. Germer, F. Chen, S. DasSarma, P. DasSarma, F. T. Robb, N. Manset, L. Kolokolova, N. Reid, F. D. Macchetto, and W. Martin, Detection of circular polarization in light scattered from photosynthetic microbes, Proc. Natl. Acad. Sci. USA, 106, 7816-7821, 2009a.

Sparks, W. B., J. H. Hough, L. Kolokolova, T. A. Germer, F. Chen, S. DasSarma, P. DasSarma, F. T. Robb, N. Manset, I. N. Reid, F. D. Macchetto, and W. Martin, Circular polarization in scattered light as a possible biomarker, J. Quant. Spectr. Radiat. Transfer, 110, 1771-1779, 2009b.

Sterzik, M. and S. Bagnulo, Polarization of leaves and life, in Astronomical Polarimetry 2008: Science from Small to Large Telescopes, edited by P. Bastien, N. Manset, D. Clemens, and N. S. Louis, ASPC series, 449, 399-401, 2011.

Toxvaerd, S., Origin of homochirality in biosystems, Int. J. Mol. Sci., 10, 1290-9, 2009.

Whitney, B. A. and M. J. Wolff, Scattering and absorption by aligned grains in circumstellar environments, Astrophys. J., 574, 205-231, 2002.

L. Nagdimunov, L. Kolokolova (e-mail: ludmilla@astro.umd.edu), and W. Sparks 